\title{
RECHERCHES EXPÉRIMENTALES \\ SUR L'ÉTIOLOGIE DE LA TULARÉMIE EN TURQUIE
}

\author{
Par SERVER KAMIL et SAIT BILAL
}

La tularémie fut découverte pour la première fois en Turquie par les médecins militaires Bican, Titiz et Kurtaran à Lüleburgaz, puis à Catalca et à Silivri. Du $1^{\text {er }}$ juillet au 7 septembre 1936 , on en compte 121 cas. En 1937, M. Talat Oz, chargé de mission par le Ministère de la Santé Publique, a trouvé plus de 35 cas nouveaux et 143 cas datant de plusieurs années, le diagnostic rétrospectif étant posé par l'agglutination et l'intradermoréaction.

Tous ces cas sont localisés à la Thrace ou Turquie d'Europe.

Pourtant, au mois d'août 1937, l'un de nous en a découvert un cas chez une jeune fille de Konya, steppe située à 1.000 mètres d'altitude, en Turquie centrale. La malade n'avait pas quitté cette région, n'avait eu aucun rapport avec la Thrace et son affection semblait bien autochtone. Nos recherches sérologiques chez les bouchers d'Ankara montrent aussi que la tularémie est plus répandue qu'on ne l'a pensé.

Le travail qui va suivre porte sur l'étiologie de la tularémie, l'étude biologique du bacille et sur quelques observations cliniques.

\section{Etiologie}

Il est intéressant de rechercher :

$1^{\circ}$ Les animaux réceptifs sauvages et domestiques qui peuvent constituer les réservoirs de virus de la maladie ;

$2^{\circ}$ Les hôtes vecteurs qui transmettent la maladie d'animal à animal et en assurent la pérennité dans la nature.

\section{$1^{\circ}$ Animaux réceptifs}

Nous étudierons successivement les animaux sauvages, les animaux domestiques et les animaux de laboratoire, car l'inoculation à l'animal constitue un moyen précieux de diagnostic.

Annales de Parasitologie, $\mathrm{T}$. XVI, $\mathrm{N}^{\circ} 6 .-1^{\text {er }}$ novembre 1938 , p. 530-542. 
A. Animaux sauvages. - Le hérisson est très sensible à la tularémie :

Après inoculation sous-cutanée, un hérisson est mort en trois jours. A l'autopsie, on a constaté un œdème au point d'inoculation, des ganglions tuméfiés, une rate augmentée de volume, un foie normal et des ecchymoses sur les poumons.

Après ingestion de viscères infectés, un hérisson meurt au bout de 5 jours avec des lésions identiques, sauf l'œè̀me local.

La gerboise est aussi sensible que le hérisson : la mort survient le $3^{\circ}$ jour après l'inoculation, ou le $5^{\circ}$ jour après ingestion d'une émulsion de culture. A l'autopsie, ón observe une congestion diffuse et quelques taches nécrotiques sur le foie.

Un blaireau inoculé sous la peau avec une culture sur milieu de Francis, le 14 janvier 1938, n'a pas semblé réceptif.

Un spermophile résiste à l'inoculation d'une très petite dose de culture. Réinjecté avec une forte dose (émulsion d'un dixième de culture), il présente un ulcère qui persiste plus de deux mois. Les ganglions ne sont pas tuméfiés. L'intradermo-réaction est positive et même nécrotique.

Un renard rouge du pays est inoculé le 7-3-38 avec une culture sous la peau : un oedème au point d'inoculation qui se transforma en une escarre et ne tarda pas à se convertir en une large plaie. Le 31-3-38, l'intradermo-réaction produit une induration de la grandeur d'une noisette; le $4-4$, sur la surface de réaction, il se forme une escarre du derme qui se transforme en une légère croûte superficielle.

Le 4-4-38, l'animal est réinoculé par voie veineuse avec une culture ; toux, polypnée après l'inoculation ; mort après 36 heures. Foie pâle et parsemé de taches hémorragiques, reins congestionnés, rate augmentée de volume, pétéchies sur le cœur et le poumon.

La caille (Coturnix communis) résiste à l'inoculation intramusculaire et à une inoculation intrapéritonéale peu abondante. L'inoculation intrapéritonéale massive tue 50 pour 100 des animaux et l'inoculation intraveineuse d'un dixième de culture les tue dans la proportion de 80 pour 100 .

Les cailles infectées par ingestion propagent le $B$. tularense par leurs matières fécales. On peut infecter des cobayes en leur faisant ingérer des excréments de caille ou en frictionnant leur peau avec ces matières.

La pintade et la perdrix sont également sensibles à l'injection intramusculaire. 
Un grand-duc (Bubo maximus) inoculé par voie intramusculaire meurt au bout de 4 jours. Ses organes inoculés à la souris lui confèrent la tularémie.

B. Animaux domestiques. - Chien. - Le 7-3-1938, une chienne est inoculée avec $1 \mathrm{~cm}^{3}$ intraveineux. L'animal meurt dans le coma le 11-3-38. Suffusion sanguine sous-cutanée, adénopathie, rate augmentée de volume et plus foncée, foie congestionné, reins couverts de pétéchies, quelques foyers de petite taille hépatisés dans le poumon gauche, taches hémorragiques sur le péricarde et congestion des méninges. La culture du sang, du foie, de la rate et de la moelle osseuse a été positive.

Une autre chienne est inoculée à la même date avec $1 \mathrm{~cm}^{3}$ sous la peau. L'animal fait une escarre au point d'inoculation. Les premiers cinq jours, l'animal présente un lumbago, balance sa croupe en marchant et boite de sa patte inoculée. Les ganglions inguinaux sont hypertrophiés.

Le 29-3-38 on lui fait une intradermo-réaction qui est positive ; au point d'inoculation on note une tache un peu surélevée, de 2,5 sur $2 \mathrm{~cm}$. de diamètre, et violacée ; le lendemain la tache devient plus foncée, le troisième jour l'épiderme se détache et laisse une plaie superficielle qui guérit en 4 jours. Le $31-3-38$, son sérum agglutine le $B$. tularense à $1 / 400$ et coagglutine le bacille de Bang à $1 / 80$. Avant l'inoculation il n'agglutinait mème à $1 / 20 \mathrm{ni}$ le $B$. tularense ni le bacille de Bang.

Le 4-4-38 nous avons réinoculé ce chien avec une culture par voie intraveineuse ; tout de suife après l'inoculation, il a vomi et montré du vertige, mais s'est vite rétabli. Le $4-4$, il boite de sa patte gauche, le 6-4, il y a du lumbago, le 7-4, de la parésie du train postérieur, le 8-4, l'animal est couché, complètement désintéressé de son entourage et ne répond pas même aux piqûres à l'aiguille ; il meurt dans la journée.

Congestion diffuse sous-cutanée, congestion générale des ganglions et adénopathie, spécialement des ganglions inguinaux et mésentériques. Vaste hémorragie sous-muqueuse de l'estomac, foie jaunâtre, friable, congestionné et hypertrophié, rate foncée et augmentée de volume, hémorragie capillaire du péricarde, lobe cardiaque du poumon gauche hépatisé, poumons et reins couverts d'ecchymoses, surrénales normales, pétéchies sur les méninges du lobe gauche du cerveau, moelle épinière normale.

Cultures du foie et de la moelle osseuse positives. 
Chat. - Quatre chats sont infectés le 24-12-37 :

Le premier reçoit sous la peau $2 \mathrm{~cm}^{3}$ d'émulsion microbienne et meurt le 31-1-38.

Le second reçoit par la même voie $5 \mathrm{~cm}^{3}$ de cette émulsion et meurt le 26-1-38.

Le troisième est inoculé avec $3 \mathrm{~cm}^{3}$ d'émulsion par voie intrapéritonéale et meurt le 27-1-38.

Le quatrième ingère $5 \mathrm{~cm}^{3}$ d'émulsion et meurt le 11-1-38.

Tous ces chats meurent dans un état de cachexie extrême. L'autopsie ne montre rien de caractéristique : le foie a un aspect marbré, la rate semble normale ; seul, le chat inoculé dans le péritoine a la rate recouverte d'un enduit fibrineux ; les ganglions du mésentère sont augmentés de volume; il n'existe pas d'autres adénopathies.

Ane. - Un âne est tué en 4 jours par inoculation intraveineuse. A l'autopsie, liquide clair séro-fibrineux dans les séreuses péritonéale et pleurale ; foie parsemé de taches jaunes et brunâtres ; rate augmentée de volume ; intestin grêle congestionné; les capsules surrénales sont aussi congestionnées et le cœur est le siège de taches pâles ; on note enfin une congestion des méninges cérébrales.

L'ingestion de cultures ne tue pas l'âne, mais son sérum agglutine le $B$. tularense à $1 / 80$ et la réaction palpébrale est positive.

Mouton. - Un mouton inoculé dans une veine meurt en 9 jours avec un foie altéré, une rate peu volumineuse et des poumons congestionnés.

Un mouton inoculé sous la peau résiste à l'infection : son sérum agglutine à 1/160 et l'intradermo-réaction laisse une zone indurée dont la persistance est longue et dépasse un mois.

Chèvre. - Une chèvre d'Ankara est tuée en 8 jours par inoculation intraveineuse. La rate est peu augmentée de volume, le foie est le siège de taches de nécrose ; des ponctuations hémorragiques sont observées sur le cœur et les surrénales.

Une autre chèvre résiste à l'inoculation sous-cutanée; mais son intradermo-réaction devient positive et son sérum agglutine à 1/80.

Réinoculée deux mois après par voie intraveineuse, cette chèvre meurt au bout de quelques heures; à l'autopsie, on trouve un liquide séro-hémorragique dans le péritoine et les plèvres; le foie est dégénéré ; la rate est macroscopiquement normale; le cœur et les poumons sont le siège d'ecchymoses.

L'ensemencement de la rate et du foie a donné des cultures de B. tularense. 
Buffle. - Cinq buffles âgés de 18 mois ont été infectés le 23-11-38 :

Deux d'entre eux reçoivent dans une veine une émulsion de culture. Ils ne mangent pas, présentent des frissons, de la toux ; la respiration est accélérée. Un des buffles guérit au bout de 4 jours, mais l'autre demeure pendant 10 jours triste, sans appétit, le dos courbé, le poil hérissé.

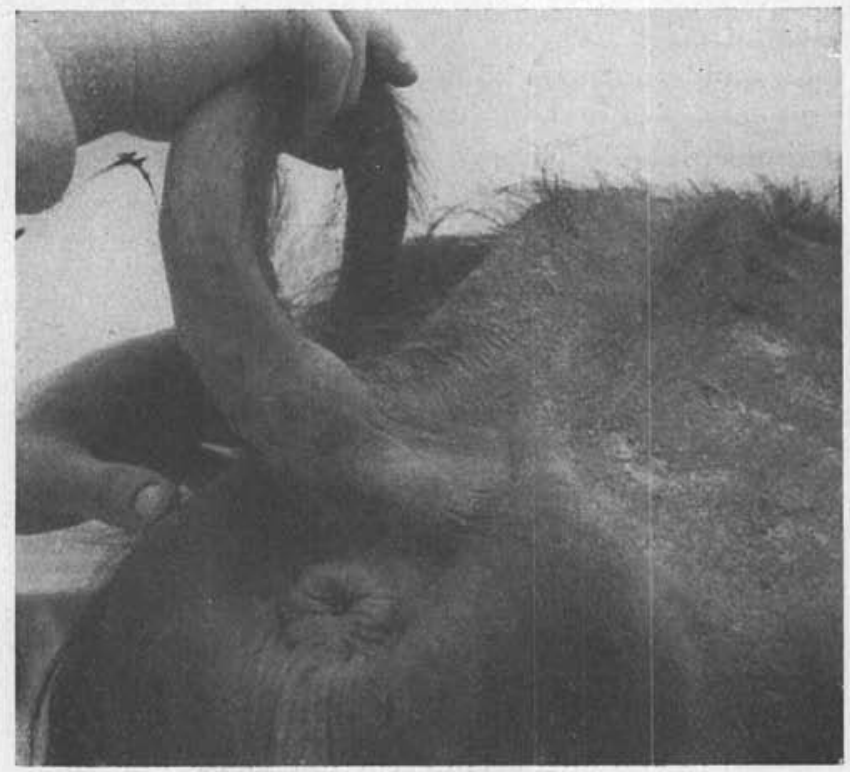

Frg. - Intradermo-réaction chez le buffle.

Deux autres buffles reçoivent sous la peau de l'encolure une culture entière de $B$. tularense. Chez ces deux animaux, les ganglions préscapulaires du côté inoculé sont augmentés de volume. L'un ne présente aucun phénomène général, tandis que l'autre ne rumine pas ; ce dernier guérit pourtant en quatre jours.

Le cinquième buffle reçoit par une sonde nasale 4 cultures de Francis en émulsion dans de l'eau salée et ne présente aucun symptôme anormal.

L'inoculation sous-cutanée à la souris de l'urine des buffles a montré leur virulence pendant plus d'un mois après l'infection.

La poule, le poussin, la dinde résistent à l'inoculation. 
Le canard, le pigeon et l'oie sont sensibles à l'inoculation intraveineuse.

Le sérum des oiseaux inoculés qui résistent à l'infection agglutine le B. tularense :

Sérum de poule : $1 / 80$; sérum de poussin : $1 / 60$; sérum d'oie : $1 / 2000$; sérum de dinde : $1 / 40$; sérum de pigeon : $1 / 320$; sérum de canard : $1 / 160$; sérum de caille : 1/160.

Le taux normal des agglutinines dans le sérum des animaux non infectés ne dépasse pas $1 / 10$.

G. Animaux de laboratoire. - La souris est l'animal le plus sensible, plus sensible même que le cobaye et le lapin domestique qui souvent résistent à l'inoculation virulente sans faire même une infection latente. Des cobayes inoculés avec le pus des ganglions sousmaxillaires de l'un de nous ont résisté même par voie intra-péritonéale, alors que des souris sont mortes au bout de 17 jours et 21 jours. L'inoculation des viscères de ces souris à deux cobayes les a tués en 4 et 5 jours.

Le cobaye peut résister à des doses plusieurs fois mortelles. Un des animaux d'expérience a fait une escarre au point d'inoculation et des adénopathies satellites ; intradermo-réaction positive ; agglutination montée à $1 / 200$. Au bout de 6 mois, le cobaye était guéri. Il fut alors réinoculé en même temps qu'un cobaye témoin. Celui-ci mourut au $5^{\circ}$ jour, tandis que le premier refaisait une infection chronique avec escarre au point d'inoculation et gonflement des ganglions.

Nỏs expériences nous autorisent à croire que l’infection tularémique n'immunise pas, elle prémunit.

Essais de transmission de la mère malade au fotus. - Une des cobayes inoculée le 16-11-1936 est morte de la tularémie le 25-111936. Elle avait trois fœtus; nous avons inoculé un cobaye avec le broyat de viscères de ces trois fœtus (le 25-11-1936). Le cobaye n'a rien montré d'anormal. L'agglutination faite les 22-12-1936, 23-11937 et 18-5-1937 était inférieure à $1 / 20^{\circ}$. L'animal est sacrifié le 19-5-1937 ; à l'autopsie nous n'avons pas vu des lésions pouvant indiquer une infection. La culture et l'inoculation des viscères sont restées négatives. De cette expérience nous pouvons conclure contre la transmission transplacentaire.

Le rat est plus résistant encore que le lapin et le cobaye. Mais on peut le tuer régulièrement avec $1 / 100^{\circ}$ de culture sous-cutanée. 


\section{2॰ Hôtes vecteurs}

Les cas de tularémie humaine observés en Europe sont généralement dus à un contact direct avec un animal malade. Aucun hôte vecteur n'a pu être incriminé jusqu'à ce jour dans la transmission de la maladie à l'homme ; mais, dans la transmission de la tularémie d'un animal à l'autre, il est plus que probable que des arthropodes parasites jouent un rôle de premier plan. Nos recherches expérimentales ont porté sur les punaises, les puces et l'Ornithodorus lahorensis.

$1^{\circ}$ Cimex lectularius. - Des punaises, nourries sur un cobaye infecté, sont placées ensuite sur des cobayes neufs. Sur les 28 cobayes en expérience, un seul a contracté la maladie : les punaises avaient été nourries sur cet animal 24 heures après le repas infectant et ensuite tous les jours pendant 16 jours.

Le cobaye était malade, mais ne présentait pas de tuméfaction ganglionnaire ; il est mort le $22^{\circ}$ jour. A l'autopsie, les ganglions étaient normaux, mais le foie et la rate étaient parsemés de nodules où la culture montra la présence de $B$. tularense.

La punaise infectée, écrasée sur la peau rasée, donne l'infection au cobaye.

Les matières fécales des punaises infectées, inoculées au cobaye, lui transmettent l'infection.

Douze larves provenant des punaises de cette expérience furent broyées, émulsionnées dans l'eau physiologique et inoculées à un cobaye par voie sous-cutanée. Le cobaye mourut un mois après, avec les lésions caractéristiques de tularémie. Remarquons qu'il ne s'agit pas sûrement d'infection héréditaire. Les larves ont pu être contaminées par des déjections des punaises adultes infectées.

$2^{\circ}$ Puces. - Vingt-six puces de chat, Ctenocephalus canis, provenant d'un chat mort de tularémie, sont broyées dans de l'eau physiologique et inoculées le 1-11-1938 sous la peau d'un cobaye. Le 14-11-38, le cobaye était encore vivant.

$3^{\circ}$ Ornithodorus lahorensis. - Ces tiques sont nourries sur un lapin infecté, puis sur un cobaye neuf pendant une heure chaque jour. Une semaine après, les ganglions inguinaux du cobaye sont déjà tuméfiés. Le sérum agglutine au $1 / 80$ au bout de 29 jours. A ce moment, on sacrifie le cobaye et l'autopsie montre des lésions caractéristiques.

Un autre cobaye est piqué par les tiques infectées tous les deux 
jours du 3-12 au 20-12 ; dès le 15-12, il existe un chapelet de ganglions. Le cobaye meurt le 24-12 avec des lésions typiques.

Ornithodorus lahorensis confère la tularémie 3 fois sur 4, à condition de piquer plusieurs fois.

Le broyat des tiques donne la tularémie au cobaye.

Le broyat de tiques mortes depuis dix jours est inoculé à un cobaye par voie sous-cutanée. Le cobaye meurt 4 jours après.

Les ornithodores avaient reçu leur dernier repas infectant quatre mois auparavant.

Toutes ces expériences sont contrôlées par la culture et l'inoculation au cobaye des lésions découvertes à l'autopsie.

\section{Etude de Quelques souches de Bacterium tularense}

A) Souches étudiées. - Nous avons poursuivi nos expériences avec 12 souches dont 6 étrangères (David, Norvège, Berlin 38, Berlin 377 , Stockholm 13, Stockholm 4) et 6 souches autochtones (Guilhane, Sait, Corlu, Ceylan, Hamzabey, Su). Toutes ces souches avaient les mêmes caractères bactériologiques, mais leur virulence était très variable; par exemple, les souches Berlin 38 et Stockholm 13 étaient presque avirulentes.

B) Milieu d'étude. - Nous avons employé un milieu de Mac Coy et Chapin, modifié par le remplacement des 40/100 d'eau physiologique par du bouillon de veau cystiné à 0,1 pour 100 et glucosé à 1 p. 100. La bactérie pousse plus abondamment et se conserve 7 mois dans un frigidaire.

Le $B$. tularense pousse très bien sur le milieu de Francis.

Nous avons obtenu des cultures sur des milieux préparés avec du sang humain et du sang de cheval, d'âne, de veau, de chèvre, de mouton, de lapin, de lièvre, de cobaye, de buffle, de poule, de dindon, de canard et d'oie.

C) Filtrabilité. - Le B. tularense traverse la bougie Berkefeld, mais non la bougie L3 Chamberland. Les cobayes inoculés avec le filtrat de L3 ne sont pas morts, mais leur sérum a agglutiné au 1/50.

D) Toxine. - Nous n'avons pas réussi à déceler la toxine, soit dans les corps microbiens, soit dans le filtrat d'une émulsion congelée 3 fois à $-14^{\circ}$ et décongelée à $37^{\circ}$. Les souris, cobayes et lapins inoculés par voie intra-veineuse avec les filtrats et les émulsions tuées ont tous résisté. 


\section{OBSERVATIONS CliniQues, RECHERCHES DIAGNOSTIQUES ET THÉRAPEUTIQUES}

A. Infections de Iaboratoire. - Après 7 mois de travail au laboratoire, l'un de nous (S. B.) s'est infecté. En ouvrant un flacon contenant de l'émulsion microbienne gelée et décongelée 3 fois de suite, une goutte a giclé dans l'œil gauche. Toutes les mesures tentées pour faire avorter l'infection ont été vaines. Après une incubation de 48 heures apparut du larmoiement; puis la conjonctivite devint manifeste avec une tendance ulcéreuse. Simultanément, les ganglions parotidiens et sous-maxillaires, d'abord sensibles à la pression, furent considérablement tuméfiés.

La maladie s'est accompagnée d'une réaction générale : fièvre élevée, frissons, vomissements, courbatures et arthralgies, en particulier au niveau des genoux.

La leucocytose était à 10.000 par $\mathrm{mm}^{3}$ et la formule leucocytaire était la suivante :

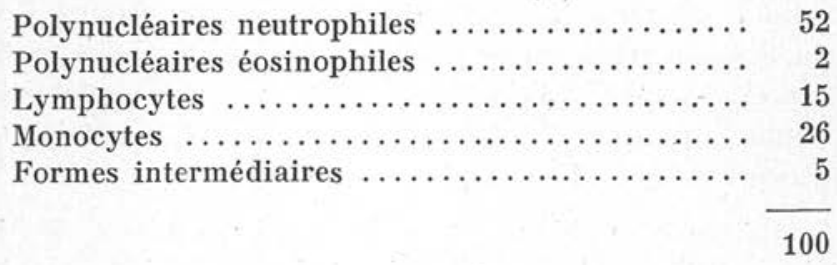

Ultérieurement, les ganglions parotidiens et sous-maxillaires évoluèrent vers la suppuration qui nécessita une intervention chirurgicale.

B. Intradermo-réaction chez l'homme et les animaux. - Une intradermo-réaction fut pratiquée sur nous-même le $35^{\circ}$ jour de la maladie.

On injecta $0 \mathrm{~cm}^{3} 1$ d'émulsion microbienne dans le derme de l'avant-bras ; 2 heures après, il existait déjà au point d'injection une papule de $1 \mathrm{~cm}$. de diamètre entourée d'un halo rosé.

Cinq heures après, cette papule rouge atteignait $2 \mathrm{~cm}$. de diamètre.

A la huitième heure, la réaction locale s'était encore étendue et une réaction générale apparut avec des frissons, de la fièvre $\left(39^{\circ} 5\right)^{-}$et des courbatures ; 24 heures après, ces signes persistaient et localement on observait un véritable placard de $10 \mathrm{~cm}$. de diamètre dont le bord était violacé et le centre commençait à se sphacéler. Les ganglions axillaires étaient tuméfiés et sensibles. 
A la $48^{\circ}$ heure, l'escarre était formée et commençait à se détacher ; la température redevint normale, tandis que l'aréole inflammatoire diminuait. La croûte tomba le $4^{\circ}$ jour, laissant une plaie rouge qui se cicatrisa en 8 jours. Parallèlement, les ganglions parotidien et sous-maxillaire diminuaient de volume, mais leur suppuration se précipita.

L'intradermo-réaction semble être un procédé de diagnostic très fidèle, mais doit être pratiquée avec certaines précautions.

Nous nous sommes servi d'une émulsion microbienne contenant 250 millions de bactéries par $\mathrm{cm}^{3}$ et chauffée pendant 2 heures à $58^{\circ}$. $1 / 10 \mathrm{de}^{\mathrm{cm}^{3}}$ de cette émulsion ne donne pas de grande réaction locale ou générale.

Chez les animaux d'expérience, l'intradermo-réaction est utile pour mettre en évidence une infection latente. Chez le cobaye, elle apparaît le $5^{\circ}$ jour de l'infection sous forme d'une petite papule rouge et dure. La réaction est d'autant plus forte que l'infection est plus avancée. Elle semble également marquée lorsque l'infection est latente.

L'intradermo-réaction chez l'animal, lorsqu'elle est intense, s'accompagne, comme chez l'homme, d'une réaction ganglionnaire et générale.

C. Sérologie. - Les propriétés agglutinantes du sérum apparaissent chez l'homme et les animaux, au cours de la $2^{\circ}$ semaine de la maladie.

Dans notre cas précité, la courbe de l'agglutination fut la suivante :

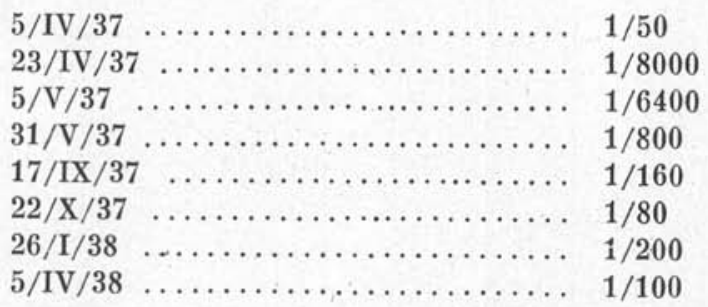

Pour se faire une idée de la fréquence de la tularémie en Turquie, nous avons recherché, avec l'aide du Service de Bactériologie, l'agglutination des souches de $B$. tularense par les sérums envoyés pour réaction de Widal et pour Wassermann. Nous n'avons eu aucun cas positif.

Ensuite, nous avons, avec l'aide du $\mathrm{D}^{\mathrm{r}}$ Suayp Barim, Directeur de 
la Santé du Vilayet d'Ankara, recherché l'agglutination chez les ouvriers de l'abattoir et les bouchers de la ville.

Sur 115 sérums éprouvés, nous avons eu 6 cas positifs :

Métiers.

Taux de l'agglutination.

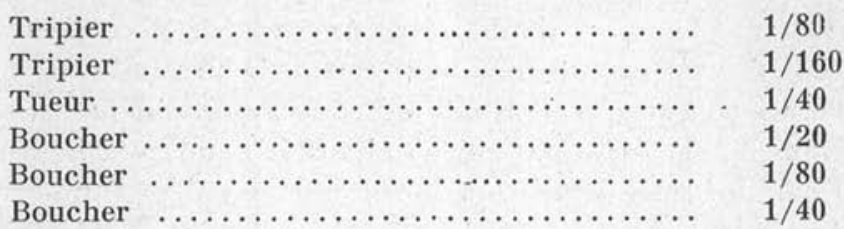

En ce qui concerne les coagglutinines, les sérums humains ne coagglutinent pas le bacille d'Eberth, les paratyphiques, le Proteus $\mathrm{X} 19$, le B. coli et les bacilles dysentériques, mais parfois les Brucella.

Les sérums d'animaux infectés, mouton, lapin, cobaye, n'agglutinent pas les Brucella. Notre sérum n'agglutina pas les Brucella tant que le taux de l'agglutination du $B$. tularense fut très élevé ; mais ensuite, le taux des coagglutinines monta à $1 / 20$.

Sur 50 sérums de malades reçus de Talat Vasfi $\mathrm{Oz}$ de Thrace, agglutinant le $B$. tularense de $1 / 10$ à $1 / 320$ et plus, plusieurs ont coagglutiné les Brucella:

$N^{\circ}$ de sérum

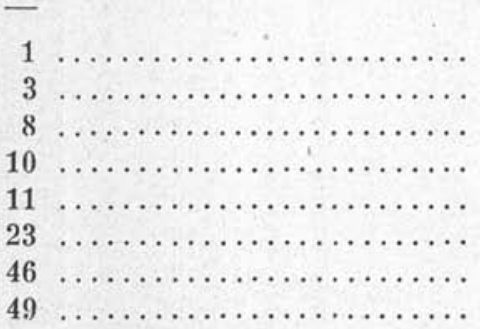

Agglutination avec

le B. tularense
Coagglutination avec le Brucella.
$1 / 320$
$1 / 320$
$1 / 320$
$1 / 320$
$1 / 320$
$1 / 10$
$1 / 40$
$1 / 10$

$1 / 20$

$1 / 80$

$1 / 20$

$1 / 80$

$1 / 20$

$1 / 40$

$1 / 20$

$1 / 20$

D. Essais de Thérapeutique. - a) Vaccination. - Elle fut tentée avec des cultures tuées et vivantes.

1. Vaccin tué. - Des cobayes sont inoculés avec une émulsion microbienne chauffée une heure à $56^{\circ}$. Ils reçoivent $1 \mathrm{~cm}^{3}, 3$ fois de suite, à une semaine d'intervalle. Une semaine après la dernière injection, on inocule une culture virulente qui tue tous les cobayes ainsi que les animaux témoins. 
2. Vaccin vivant. - Nous avons employé la souche Stockholm 13, qui ne tue pas le cobaye. Le 14-10-37, 12 cobayes sont inoculés sous la peau avec $1 \mathrm{~cm}^{3}$ de cette culture. Un œdème passager se forme au point d'inoculation et les ganglions inguinaux augmentent de volume. L'intradermo-réaction est positive. Le 8-12-37, ces cobayes sont inoculés, ainsi que deux témoins, avec une culture virulente. Les témoins meurent au bout de 7 à 11 jours.

Les cobayes vaccinés ont fait un abcès au point d'inoculation et des adénites, mais ils ont résisté. Les ganglions diminuèrent de volume, mais l'intradermo-réaction était toujours positive.

Le 23-11-37 et le 8-12-37, nous avons sacrifié deux cobayes. A l'autopsie, les ganglions contenaient un pus caséifié jaunâtre; le foie et surtout la rate étaient parsemés de nodules.

On doit, semble-t-il, parler ici de prémunition plus que d'immunisation.

Signalons en outre qu'un des cobayes vaccinés a mis bas le 16-10-37 alors que son intradermo-réaction était encore positive. Le petit cobaye avait une intradermo-réaction négative le 4-11-37 et le 12-1-38, mais le 4-2-38, son sérum agglutinait à $1 / 320$ et le 29-2-38 l'intradermo-réaction devenait positive. Il a maintenant 4 mois et ne présente aucun symptôme anormal.

b) Sérothérapie. - Nous avons inoculé 2 moutons avec une émulsion de microbes tués et enrobés dans de la lanoline. L'émulsion contenait 20 milliards de bactéries par $\mathrm{cm}^{3}$.

\begin{tabular}{|c|c|c|c|}
\hline Dates & Quantité d' & émulsion & Quantité de mé \\
\hline - & & & - \\
\hline $5 / \mathrm{V} / 37$ & & $\mathrm{~cm}^{3}$ & $2 \mathrm{~cm}^{3}$ \\
\hline $14 / V / 37$ & $\ldots \ldots \ldots \ldots \ldots \ldots$ & - & $5-$ \\
\hline $28 / \mathrm{V} / 37$ & $\ldots \ldots \ldots \ldots \ldots \ldots$ & - & $10-$ \\
\hline $15 / \mathrm{VI} / 37$ & ........... & - & $20-$ \\
\hline $30 / \mathrm{VI} / 37$ & $\ldots \ldots \ldots \ldots \ldots$ & - & $40-$ \\
\hline $15 / \mathrm{VII} / 37$ & $\ldots \ldots \ldots \ldots \ldots$ & - & $80-$ \\
\hline $30 / \mathrm{VII} / 37$ & $\ldots \ldots \ldots \ldots \ldots 12$ & - & $120-$ \\
\hline
\end{tabular}

Le sérum prélevé une semaine après la dernière injection agglutinait le $B$. tularense à $1 / 1.800$ pour le premier mouton et à $1 / 1.600$ pour le second. Le mélange des sérums des deux animaux n'avait aucun pouvoir curatif ni préventif sur des souris. Les souris témoins -sont mortes en 4 jours et les souris inoculées en 6 ou 7 jours, c'est-à-dire avec un certain retard. 
c) Ghimiothérapie. - Nous avons fait cette expérience avec le $\mathrm{D}^{\mathrm{r}}$ Ali Mustafa, en employant le prontosil et le paramino benzène sulfamide.

$1^{\circ}$ On fait ingérer 3 jours de suite 0 gr., 01 de médicament, et ensuite, on injecte $0 \mathrm{~cm}^{3}$, 5 de l'émulsion virulente qui tue les souris.

$2^{\circ}$ Les souris sont inoculées d'abord, et 24 heures après, ingèrent le médicament. Ces souris meurent.

\section{Conclusions}

$1^{\circ}$ Les recherches de Talat $\mathrm{Oz}$ en Thrace et nos constatations personnelles nous font croire que la maladie existait depuis des années en Turquie.

$2^{\circ}$ Le nombre des mammifères et des oiseaux, sauvages et domestiques, sensibles à la tularémie est très grand. Les animaux infectés éliminent les bactéries par la bile et les urines; ceci peut expliquer l'infestation massive d'un cours d'eau, surtout en été, par les urines de gros animaux, buffles par exemple.

$3^{\circ}$ La transmission de la maladie est possible par les punaises et l'Ornithodorus lahorensis, argasiné très répandu en Turquie.

$4^{\circ}$ Le diagnostic de la tularémie peut être fait par l'inoculation à la souris que nous préférons au cobaye, par l'intradermo-réaction et le séro-diagnostic.

$5^{\circ}$ Il ne semble pas exister d'immunité dans la tularémie, mais une prémunition.

$6^{\circ}$ La vaccination, la sérothérapie, la chimiothérapie se sont jusqu'à ce jour montrées inefficaces.

\section{Bibliographie}

Asim Arar. - Off. Intern. Hyg. publique, $\mathrm{n}^{\circ}$ 9, sept. 1937.

Kemal Hüseyin. - Pratik doktor, n 9, p. 129, sept. 1936.

- Tedavi klinigi ve laboratuvar Dergisi, $\mathrm{n}^{\circ}$ 7, p. 109, sept. 1937.

- Zeitschr. f. Hyg., CXIX, 1937.

S. Kамп Токаӧz. - Sihhige mecmuasi, XIII, n ${ }^{\circ} 58,1937$.

O. Bican, I. Titiz et M. F. Kurtaran. - Tularémie, Monographie, 1936.

S. Brlal. - Türk Baytarlar Birligi Dergisi, VII, n* 5-6, 1937.

TAlat VASFi Oz. - Zentralb. f. Bakter (Referate) (B), CXXIX, n ${ }^{\circ s}$ 5-6, 1938.

Institut d'Hygiène d'Ankara. 